\title{
Suppression of graft-versus-host disease and retention of graft-versus-tumour reaction by murine genetically engineered dendritic cells following bone marrow transplantation
}

\author{
YIHONG HUANG $^{1,2^{*}}$, SARAN FENG $^{3 *}$, YUJIE XU ${ }^{1,2}$, WANRU CHEN ${ }^{1,2}$, SHUHUA WANG $^{1}$, \\ DEPENG LI ${ }^{1}$, ZHENYU LI ${ }^{1}$, QUNXIAN LU ${ }^{1}$, XIUYING PAN ${ }^{1}$ and KAILIN XU ${ }^{1}$ \\ ${ }^{1}$ Department of Hematology, The Affiliated Hospital of Xuzhou Medical College, Xuzhou, Jiangsu 221002; \\ ${ }^{2}$ Department of Hematology, The Third Affiliated Hospital of Xuzhou Medical College, Xuzhou, Jiangsu 221003; \\ ${ }^{3}$ Department of Hematology, Shandong Provincial Qianfoshan Hospital, Jinan, Shandong 250014, P.R. China
}

Received November 27, 2013; Accepted September 18, 2014

DOI: $10.3892 / \mathrm{mmr} .2014 .3123$

\begin{abstract}
The effect of infusion of lentiviral vector-mediated, genetically engineered dendritic cells (DCs) following allogeneic bone marrow transplantation (allo-BMT) on graft-versus-host disease (GVHD) and graft-versus-leukemia (GVL) was investigated in a mouse model.Lentivirus-mediated expression of soluble tumor necrosis factor receptor 1 (sTNFR1) converted immature DCs (imDCs) from BABL/c mice into engineered DCs in vitro. An EL4 leukemia allo-BMT model of BABL/c to C57BL/6 mice was established. Engineered DCs with donor bone marrow cells and splenocytes were subsequently transplanted into myeloablatively irradiated recipients. The average survival duration in the sTNFR1- and pXZ9-imDC groups was significantly prolonged compared with that of the allo-BMT group $(\mathrm{P}<0.05)$. Mild histological changes in GVHD or leukemia were observed in the recipients in the sTNFR1-imDC group and clinical GVHD scores in this group were significantly decreased compared with those of the transplantation and pXZ9-imDC groups. Serum interferon- $\gamma$ levels were decreased in the pXZ9-imDC and sTNFR1-imDC groups compared with those in the allo-BMT group $(\mathrm{P}<0.05)$, with the reduction being more significant in the sTNFR 1-imDC group $(\mathrm{P}<0.05)$. Serum interleukin-4 expression levels were decreased in the allo-BMT group, but gradually increased in the pXZ9-imDC and sTNFR1-imDC groups $(\mathrm{P}<0.05)$.
\end{abstract}

Correspondence to: Professor Yihong Huang or Professor Kailin Xu, Department of Hematology, The Affiliated Hospital of Xuzhou Medical College, 99 Huaihai Road West, Xuzhou, Jiangsu 221002, P.R. China

E-mail: yihonghuangcn@126.com; hxr1583@sina.com

E-mail: kailinxu@126.com

*Contributed equally

Key words: dendritic cells, transgenes, allograft, transplantation, immunotolerance, antileukemia
Co-injection of donor genetically-engineered imDCs was able to efficiently protect recipient mice from lethal GVHD while preserving GVL effects during allo-BMT.

\section{Introduction}

The therapeutic efficacy of allogeneic bone marrow transplantation (allo-BMT) for the treatment of a variety of neoplastic diseases, including hematological malignancies, relies upon the graft-versus-tumor (GVT) effects that eliminate residual malignant cells. To date, acute graft-versus-host disease (GVHD) remains the most severe complication of allo-BMT, which limits its application and efficacy $(1,2)$. Therefore, minimizing the incidence and severity of GVHD without sacrificing critical GVT activity remains a major clinical challenge for bone marrow transplantation (BMT). Therapeutic approaches developed to attenuate GVHD have focused on the production of immunosuppressive agents and the ex vivo removal of donor T cells from bone marrow grafts. However, T-cell depletion is associated with increased rates of engraftment failure, sustained immunosuppression and leukemia relapse. Therefore, more effective therapies are required in order to reduce the incidence and severity of GVHD and improve the survival rate of transplant recipients. Strategies for the reliable induction of a robust, permanent state of immunological tolerance may markedly improve the prospects of allograft recipients. Strategies for inducing immunologic tolerance in hematopoietic stem cell transplantation may be able to decrease or prevent the incidence of GVHD (3-5). Acute GVHD fundamentally depends upon donor T-cell interaction with antigen-presenting cells (APC) and their subsequent activation, proliferation and differentiation, a process which occurs during the second step of the afferent phase.

Dendritic cells (DCs) represent a heterogeneous population of professional APCs. DCs have crucial functions in the initiation and regulation of immune responses and are additionally involved in mediating the induction and maintenance of immune tolerance. The ability of DCs to initiate immune responses or induce immune tolerance is dependent on their maturation status. DCs are altered immunophenotypically 
and functionally during maturation. Normal immature DCs (imDCs) that lack co-stimulatory molecules, including CD80 and CD86, exhibit tolerance-inducing activities, but demonstrate immunogenicity following maturation $(6,7)$. However, the clinical applications of normal imDCs may be unsuitable for the treatment of immunopathogenic diseases, as they may mature under inflammatory conditions (8). Therefore, the prevention of DC maturation or the maintenance of imDC in their immature state is required for the induction of long-term immune tolerance in vivo $(9,10)$. Tumor necrosis factor $\alpha$ (TNF- $\alpha)$, which is a potent pro-inflammatory cytokine produced by stimulated monocytes, macrophages, activated T cells and DCs, is a critical mediator of alloreactive responses (11). The development of DCs from progenitor cells to mature DCs that possess potential immune response functions requires multiple cytokines, including TNF- $\alpha(12,13)$. TNF- $\alpha$ is important in DC differentiation and maturation. Inhibition of TNF- $\alpha$ function may arrest DCs in an immature state, prolonging their tolerogenic potential $(14,15)$. TNF- $\alpha$ binds to two independent cell surface receptors, type 1 (p55) and type 2 (p75); TNF receptor 1 (TNFR1) is the major signal transducer (16). Soluble TNFR1 (sTNFR1) and sTNFR2, which are formed by shedding the extracellular domain of TNFR, are effective in blocking and neutralizing TNF- $\alpha(17,18)$. However, whether or not the blockade of TNF- $\alpha$ by sTNFR1 is able to inhibit the maturation of DCs and potentiate imDC tolerogenicity remains to be elucidated. In a previous study by our group, a recombinant lentiviral vector expressing sTNFR1 was constructed and successfully used to transfect imDCs, which maintained a long-term immature status. The immunological characteristics of the sTNFR1 gene-modified imDCs were additionally analyzed (19). In the present study, mouse leukemia models were established in order to investigate whether sTNFR1-induced tolerogenic imDCs were able to reduce the effects of acute GVHD following allo-BMT whilst maintaining graft-versus-leukemia (GVL) responses to provide a novel approach for potential therapeutic application.

\section{Materials and methods}

Animals and cell strains. Female C57BL/6 mice $\left(\mathrm{H}-2^{\mathrm{b}}\right)$ were used as recipients and male BALB/c mice $\left(\mathrm{H}-2^{\mathrm{d}}\right)$ were used as donors. All animals, aged eight to 12 weeks, weighing 18 to $22 \mathrm{~g}$, were provided by the Laboratory Animal Centre of Yangzhou University (Yangzhou, China). Mice were reared in cages under a $12 \mathrm{~h}$ light/dark cycle at a constant temperature $\left(25 \pm 2^{\circ} \mathrm{C}\right)$ and $50-60 \%$ relative humidity. Mice were administered sterile water containing $250 \mathrm{mg} / \mathrm{l}$ erythromycin (Xi'an Lijun Pharmaceutical Co., Ltd., Xi'an, China) and $320 \mathrm{mg} / 1$ gentamicin (Chengdu Haitong Pharmaceutical Co., Ltd., Chengdu, China) for seven days prior to transplantation and were transferred to a fume cupboard following transplantation. The food and pads were sterilized. The lymphoma cell-strain EL4 was purchased from the Cell Bank of Shanghai Institute of Cell Biology (Shanghai, China) and was used as the T-cell leukemia/lymphoma cell line in C57BL/6 mice. The present study was performed in accordance with the recommendations in the Guide for the Care and Use of Laboratory Animals of the National Institutes of Health. The experimental animal protocol was reviewed and approved by the Institutional Animal Care and Use Committee of the Affiliated Hospital of Xuzhou Medical College (Xuzhou, China).

Separation of bone marrow and spleen cells. The bone marrow mononuclear cell suspension from BALB/c mice was collected under sterile conditions (20) and adjusted to a cell concentration of $5 \times 10^{7}$ cells $/ \mathrm{ml}$. BALB/c mouse spleens were harvested to prepare a spleen cell suspension. Mononuclear cells were obtained using lymphocyte isolation solution (Shenzhen Dakewe Biotech Co., Ltd., Shenzhen, China) and the cell concentration was adjusted to $5 \times 10^{7}$ cells $/ \mathrm{ml}$. Cell viability was $>95 \%$, as detected by trypan blue staining.

Construction of lentivirus. Reconstruction, identification and packaging of lentiviral vectors were performed as described previously (19). The correctly ligated recombinant plasmid was named pXZ208-sTNFRl-IRES-enhanced green fluorescence protein (eGFP) (pXZ9-sTNFRl) using liposome Lipofectamine ${ }^{\mathrm{TM}}$ reagent (Invitrogen Life Technologies, Carlsbad, CA, USA). Plasmids pXZ9-sTNFR1, pXZ9, $\Delta$ NRF and envelope protein plasmid VSVG (all obtained from the Laboratory of Hematology, The Affiliated Hospital of Xuzhou Medical College, Xuzhou, China) were co-transfected into 293 FT cells (Laboratory of Hematology, Affiliated Hospital of Xuzhou Medical College) in the exponential growth phase. At 24 and $48 \mathrm{~h}$ following transfection, eGFP expression was observed under an IX71 fluorescence microscope (Olympus Optical Co., Ltd., Tokyo, Japan). At 48, 72 and 96 h following transfection, the virus containing the supernatant was collected, condensed, filtered and preserved at $-80^{\circ} \mathrm{C}$ for further use.

imDC culture and transfection. Bone marrow suspension was collected from BALB/c mice, placed in six-well culture plates at $1 \times 10^{6}$ cells per well and incubated in RPMI-1640 (Gibco-BRL, Invitrogen Life Technologies, Carlsbad, CA, USA) containing $20 \mu \mathrm{g} / \mathrm{l}$ granulocyte-macrophage colony-stimulating factor (GM-CSF) and $10 \mu \mathrm{g} / 1$ interleukin-4 (IL-4) (PeproTech, Inc., Rocky Hill, NJ, USA) in a 5\% $\mathrm{CO}_{2}$ incubator at $37^{\circ} \mathrm{C}$. Cell growth was monitored using a CKX41 inverted phase contrast microscope (Olympus Optical Co., Ltd.). Following $48 \mathrm{~h}$ of culture, the culture media and any freely floating cells were discarded and the attached cells were cultured in fresh RPMI-1640 medium (Gibco-BRL, Invitrogen Life Technologies) containing cytokines at the same concentration for five days. Then $10 \mathrm{~g} / \mathrm{l}$ exogenous lipopolysaccharide was used to stimulate the cells, followed by culture until day 7. Cells were harvested, incubated with CD16/CD32 monoclonal antibodies (BioLegend Inc., London, UK) at $4{ }^{\circ} \mathrm{C}$ for 30 min to block Fc receptors and subsequently incubated with anti-mouse fluorescein isothiocyanate (FITC)-CD40, CD80, phycoerythrin (PE)-CD86, CD11c and FITC-I-A/I-E monoclonal antibodies (BioLegend, Inc., London, UK) for $30 \mathrm{~min}$. Phenotyping was performed using flow cytometry (FACSCalibur flow cytometer; Becton, Dickinson and Company, East Rutherford, NJ, USA). The imDCs cultured for five days were collected, placed in fresh culture medium containing GM-CSF and IL-4 and subsequently cultured with 

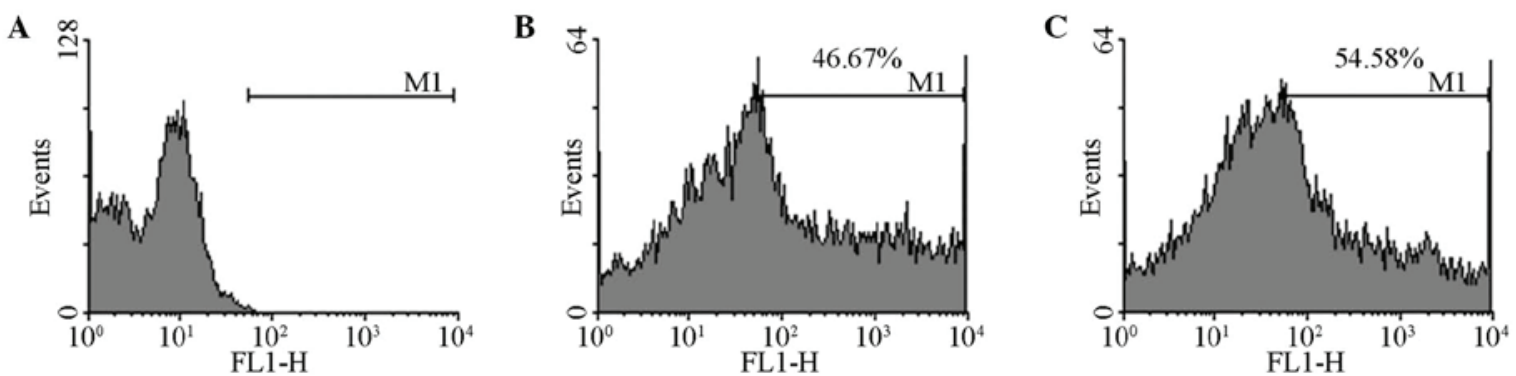

Figure 1. Infection efficiency of dendritic cells transduced with (A) no virus; (B) pXZ9 lentivirus or (C) soluble tumor necrosis factor receptor 1 lentivirus. FL1-H represents green fluorescent protein-H quantified by flow cytometry.

$5 \mathrm{ml}$ pXZ9-sTNFR1 or pXZ9 virus (empty vector control) to generate sTNFR1-imDC or pXZ9-imDCs, followed by polybrene (Sigma-Aldrich Inc., Seelze, Germany) to achieve a concentration of $8 \mathrm{mg} / \mathrm{l}$. Following viral infection for $96 \mathrm{~h}$, cells were harvested and eGFP expression was observed using a fluorescence microscope. Viral infection efficiency was determined using flow cytometry. DC surface markers were analyzed using flow cytometry. Gene transcription of sTNFR1 in target cells was assessed using reverse transcription polymerase chain reaction (RT-PCR). The sTNFR1 protein levels in cell supernatants were determined by western blot analysis (19).

EL4 leukemia/lymphoma model and grouping. On the day of transplantation, donor mice underwent X-ray total body irradiation (TBI) using a linear accelerator $(7.5 \mathrm{~Gy}$ in total at a rate of $0.5 \mathrm{~Gy} / \mathrm{min}$ ). Transplantation was performed $4 \mathrm{~h}$ following irradiation. Mice were randomly assigned to five groups $(n=10$ per group), excluding those used for chimera analysis and cytokine determination: $i$ ) TBI alone, infused with $0.3 \mathrm{ml}$ normal saline by the caudal vein; $i$ ) leukemia model group, infused with isogeneic $5 \times 10^{6}$ spleen cells, $5 \times 10^{6}$ bone marrow cells and $5 \times 10^{3}$ EL 4 cells by the caudal vein; iii) allo-BMT group, infused with donor mice $5 \times 10^{6}$ spleen cells, $5 \times 10^{6}$ bone marrow cells and $5 \times 10^{3}$ EL 4 cells by the caudal vein (data for the GVHD model were excluded because the GVHD model was common $(4,20)$ and a GVL effect was observed); iv) pXZ9-imDC group, infused with $5 \times 10^{6}$ spleen cells from donor mice, $5 \times 10^{6}$ bone marrow cells, $5 \times 10^{3}$ EL4 cells and $5 \times 10^{6}$ pXZ9-imDCs by the caudal vein; and v) sTNFR1-imDC group, infused with donor mice $5 \times 10^{6}$ spleen cells, $5 \times 10^{6}$ bone marrow cells, $5 \times 10^{3}$ EL4 cells and $5 \times 10^{6}$ sTNFR1-imDCs by the caudal vein.

Observation parameters. Food intake and behavior were assessed daily following transplantation. Survival duration was recorded to calculate the survival rate. Peripheral blood cells were quantified regularly and blood film was examined to evaluate hematogenesis. A white blood cell count of $<0.5 \times 10^{9} / 1$ represented transplantation failure, whereas a count of $>1.0 \times 10^{9} / 1$ represented hematopoietic recovery. GVHD was diagnosed in mice exhibiting a white blood cell count of $>10 \times 10^{9} / 1$, lethargy, reduced activity, weight loss, hunched back, ruffled fur and diarrhea. GVHD severity was evaluated based on weight loss, activity, posture, ruffled fur and presence of dermal lesions (for a total score of 10) (21). The GVL effect was evaluated according to mouse survival duration, survival rate, mixed chimera formation and peripheral blood examination. Leukemia-induced mortality was identified by a white blood cell count of $>20 \times 10^{9} / 1$, enlarged liver and spleen and a large number of leukemia cells in the peripheral blood. In contrast to leukemia- and GVHD-induced mortalities, mortality caused by hematopoietic depression-induced infection and hemorrhaging within two weeks of transplantation was defined as transplantation-associated mortality.

Histopathological examination. The liver, spleen, small intestine and skin were collected from moribund mice with GVHD, or recipients sacrificed at $>30 \mathrm{~d}$, in each group. Tissues were sliced, fixed with $100 \mathrm{~g} / \mathrm{l}$ formaldehyde (Shanghai Haling Biological Science and Technology Co., Ltd., Shanghai, China), embedded in paraffin, and stained with hematoxylin and eosin (Boster Biological Engineering Co., Ltd., Wuhan, China). Sections were observed with an SZ61 optical microscopy (Olympus Optical Co., Ltd.) to detect pathological GVHD changes and leukemia cell infiltration $(22,23)$.

Peripheral blood cytokine determination following transplantation. Blood was harvested from the eyeballs of recipient mice in the allo-BMT, pXZ9-imDC and sTNFR1-imDC groups at $0,7,14,21$ and $28 \mathrm{~d}$ following transplantation and stored at $-80^{\circ} \mathrm{C}$. IL-4 and interferon (IFN) $\gamma$ concentrations in the serum were determined using ELISA kits (Genetimes Technology Co., Ltd., Shanghai, China).

Chimera detection. Bone marrow cells $\left(1 \times 10^{6}\right)$ were harvested from recipient mice surviving $>30 \mathrm{~d}$, incubated with PE-labelled anti-H-2Kb monoclonal antibody (BD Pharmingen, San Diego, CA, USA) for $20 \mathrm{~min}$ in the dark, treated with hemolysin for $5 \mathrm{~min}$ in the dark and washed with phosphate-buffered saline. The percentage of cells from donor mice was determined using flow cytometry and allogeneic chimeras were detected using Cellquest software version 3 (BD Biosciences, Franklin Lakes, NJ, USA).

Statistical analysis. Values are expressed as the mean \pm standard deviation and analyzed using SPSS 16.0 software (SPSS, Inc., Chicage, IL, USA) with one-way analysis of variance. Paired comparisons among groups were conducted using the q-test. Survival curves were calculated using the Kaplan-Meier method and compared using the log-rank test. The leukemia incidence was determined using the $\chi^{2}$ test. A value of $\mathrm{P}<0.05$ 
A

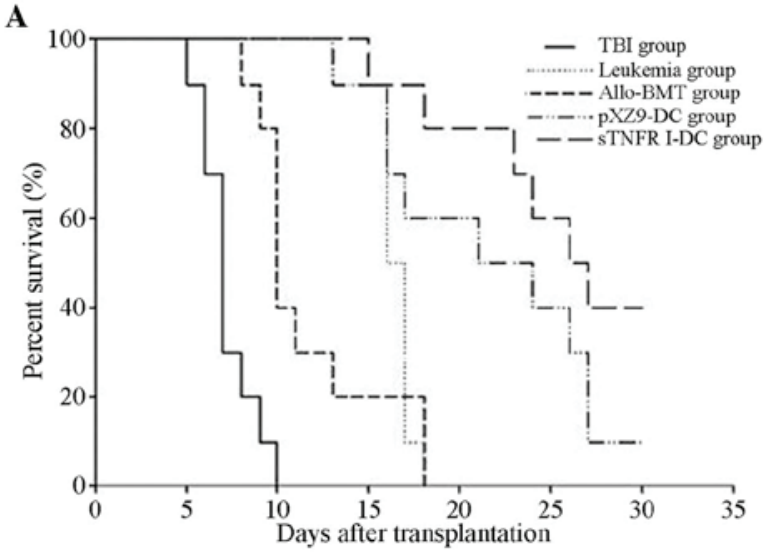

B

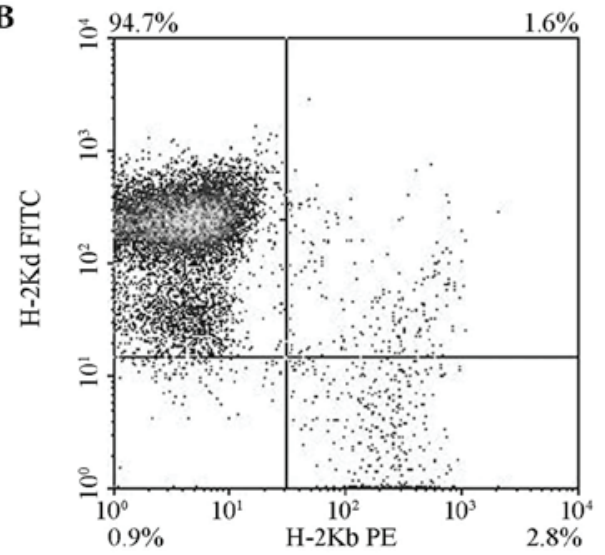

Figure 2. Survival rate of each group and chimerism of recipient animals that survived for 30 d following transplantation. (A) Survival rate of each group following transplantation; (B) Chimerism of recipient animals that survived for $30 \mathrm{~d}$ following transplantation. Bone marrow cells were dual-fluorescence stained with PE-labelled anti-H-2 ${ }^{\mathrm{b}}$ monoclonal antibody and FITC-labelled anti-H-2 ${ }^{\mathrm{d}}$ monoclonal antibody. FITC, fluorescein isothiocyanate; PE, phycoerythrin; sTNFR1, soluble tumour necrosis factor receptor 1; allo-BMT, allogeneic bone marrow transplantation; DC, dendritic cell.

was considered to indicate a statistically significant difference between values.

\section{Results}

Lentiviral infection of imDCs. Mouse bone marrow mononuclear cells adhered following $24 \mathrm{~h}$ of culture. Cell colonies were observed for 2-3 d and gradually increased in number and size. Following $5 \mathrm{~d}$ of culture, variously sized protuberant burr formed on the surface of certain cells, and single floating DCs were observed. DCs at $5 \mathrm{~d}$ were positive for CD11c and negative for CD40, CD86, CD80 and major histocompatibility complex (MHC) II. Following 6-8 d of culture, various sizes of protuberant burrs formed on the surface of certain cells and singular floating cells displaying typical DC appearance were observed. At $7 \mathrm{~d}$ following lipopolysaccharide stimulation, imDCs or pXZ9-imDCs expressed MHC II, CD40, CD80 and CD86, indicating mature DC status. By contrast, MHC II, CD40, CD80 and CD86 expression remained unchanged in the sTNFR1-imDC group. sTNFR1- or pXZ9-packaged recombinant lentiviruses were used to infect imDCs. Fluorescence microscopy observation detected eGFP expression in half of the cells, which was consistent with the flow cytometry results (Fig. 1). RT-PCR indicated a specific band that was $368 \mathrm{bp}$ in size in imDCs injected with lentivirus carrying the sTNFR1 gene, but not in that of the control group. Western blot analysis detected sTNFR1 protein in the supernatant of lentivirus-infected imDCs, but not in that of the control group.

Survival of mice following transplantation. Mice from the TBI alone group died between six and ten days following transplantation. The average survival duration was $7.8 \pm 1.3 \mathrm{~d}$. Mice from the leukemia model group succumbed to leukemia 13-18 d following transplantation. The average survival duration was $16.60 \pm 0.97 \mathrm{~d}$. The first mice from the allo-BMT group died eight days following transplantation and all mice had died by day 18 . The majority of the mice in the pXZ9-imDC group died within $21 \mathrm{~d}$, although $10 \%$ of the mice survived for $>30 \mathrm{~d}$. The average survival duration was $21.70 \pm 5.80 \mathrm{~d}$, which was significantly prolonged in comparison with the survival duration of the allo-BMT group $(\mathrm{P}<0.05)$. Mice in the sTNFR1-imDC group first died at day 15 and $40 \%$ of the mice survived up to $30 \mathrm{~d}$. The average survival duration was $25.80 \pm 5.20 \mathrm{~d}$, which was significantly prolonged in comparison with the survival duration of the allo-BMT group $(\mathrm{P}<0.05)$ and was also longer than that of the pXZ9-imDC group ( $\mathrm{P}>0.05$; Fig. 2A). Flow cytometric analysis of bone marrow cells from mice surviving for $>30 \mathrm{~d}$ in the allo-BMT, pXZ9-imDC and sTNFR1-imDC groups revealed that $95-100 \%$ of cells were $\mathrm{H}-2^{\mathrm{d}}$ positive, demonstrating complete donor chimerism (Fig. 2B).

GVHD incidence. Acute GVHD clinical manifestations, including lethargy, reduced activity, weight loss, hunched back, ruffled fur, diarrhea and hair loss, were observed in the allo-BMT, pXZ9-imDC and sTNFR1-imDC groups. Symptoms were most severe in the allo-BMT group, with a clinical score of $6.8 \pm 1.2$. The GVHD scores in the pXZ9-imDC and sTNFR1-imDC groups were 5.5 \pm 1.1 and $4.2 \pm 1.0$, respectively. These values were significantly lower than scores in the allo-BMT and pXZ9-imDC groups $(\mathrm{P}<0.05)$ (Fig. 3A). Pathological examination indicated that the GVHD pathological grade of the skin, liver and small intestine was III-IV in the allo-BMT group; II-III in the pXZ9-imDC group and 0-I in the sTNFR1-imDC group (0-I, mild; II-III, moderate; III-IV, severe; Fig. 3B).

Leukemia incidence. Mice in the leukemia model group all succumbed to leukemia within $18 \mathrm{~d}$. Histopathological examination revealed infiltration by a large number of leukemia cells (Fig. 4), with a $20 \times 10^{9} / 1$ to $24 \times 10^{9} / 1$ white blood cell count in peripheral blood. The incidence of leukemia in the allo-BMT, pXZ9-imDC and sTNFR1-imDC groups was 10, 20 and $10 \%$, respectively. No statistically significant difference was observed between the groups $(\mathrm{P}>0.05)$.

Cytokine expression alterations in mice following transplantation. The serum IFN- $\gamma$ concentration peaked at $14 \mathrm{~d}$ in the allo-BMT, pXZ9-imDC and sTNFR1-imDC groups and subsequently decreased. It was significantly reduced in the sTNFR1-imDC group compared with that of the allo-BMT and 
A

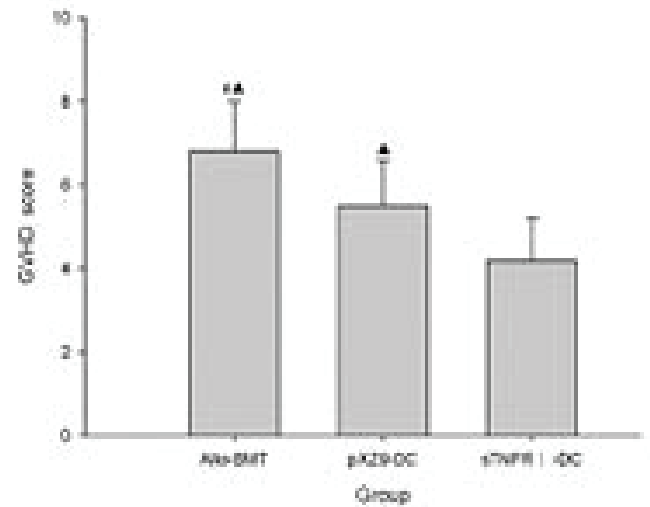

B

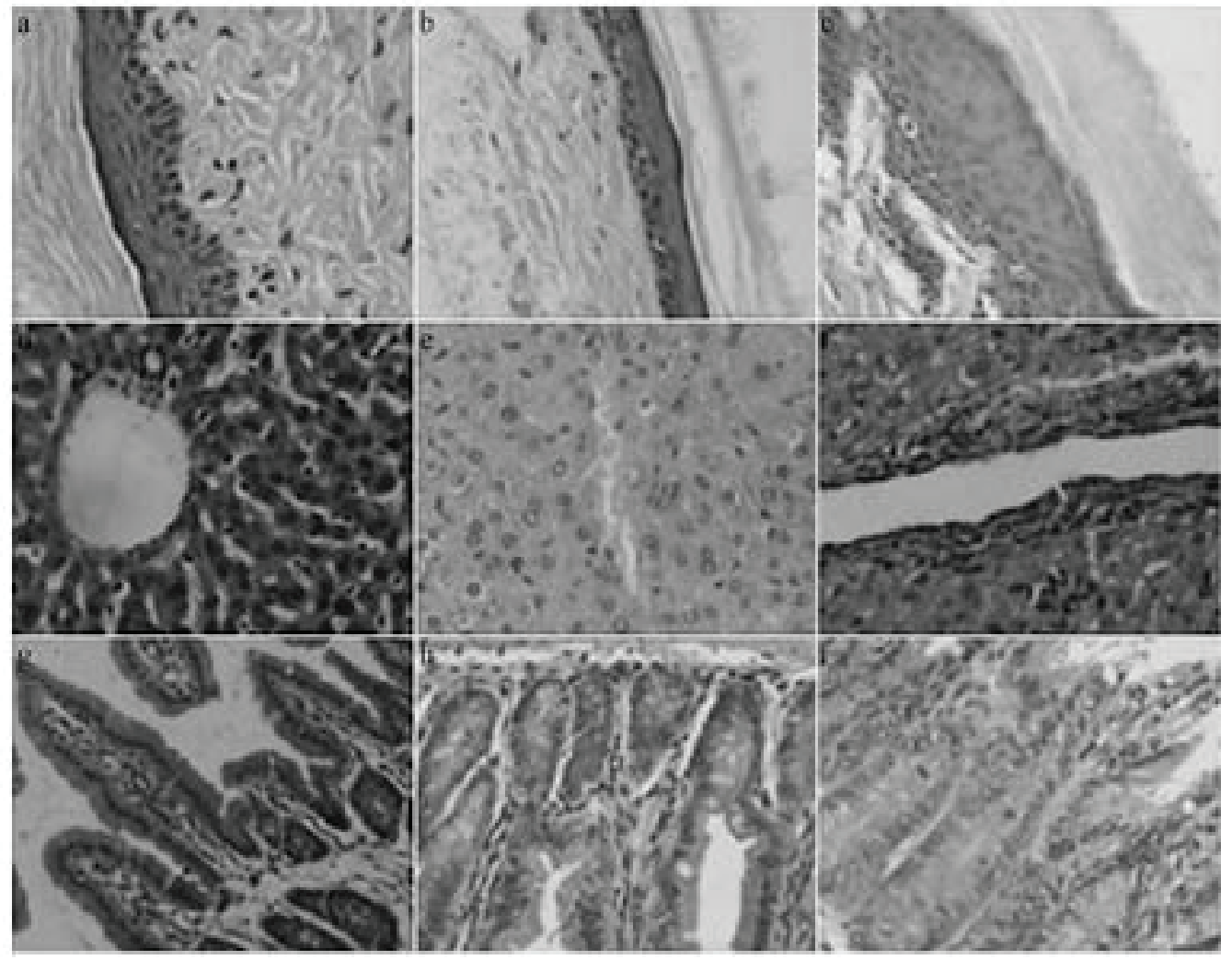

Figure 3. Clinical score of GVHD and histopathological evidence in the liver, small intestine and skin of mice following transplantation. (A) Average total GVHD score of groups at serial time-points following transplantation. (B) Histopathological evidence in liver, small intestine and skin of mice following transplantation. (a, d and g) Skin, liver and small intestines of mice which exhibited no evidence of GVHD in the sTNFR1-imDC group; (b, e and h) skin, liver and small intestines of mice with moderate GVHD in the pXZ9-imDC group; (c, $f$ and i) skin, liver and small intestines of mice with severe GVHD in the allo-BMT group. ( ${ }^{\#} \mathrm{P}<0.05$ vs. pXZ9-imDC group; ${ }^{\wedge} \mathrm{P}<0.05$ vs. sTNFR1-imDC group, hematoxylin and eosin staining; magnification, $\left.\mathrm{x} 400\right)$. GVHD, graft-versus-host disease; allo-BMT, allogeneic bone marrow transplantation; imDC, immature dendritic cell; sTNFR1, soluble tumour necrosis factor receptor 1.

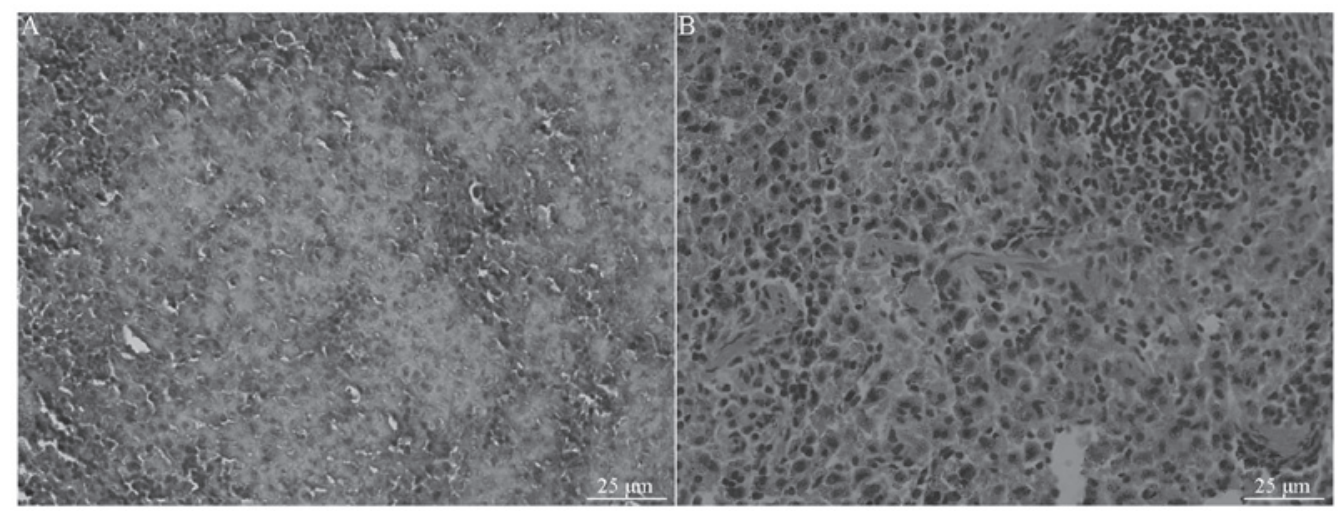

Figure 4. Spleen pathology of leukemic mice. (A) Spleen without leukemia cells. (B) Spleen with leukemia cells (hematoxylin and eosin staining; magnification, $\mathrm{x} 400$ ). 

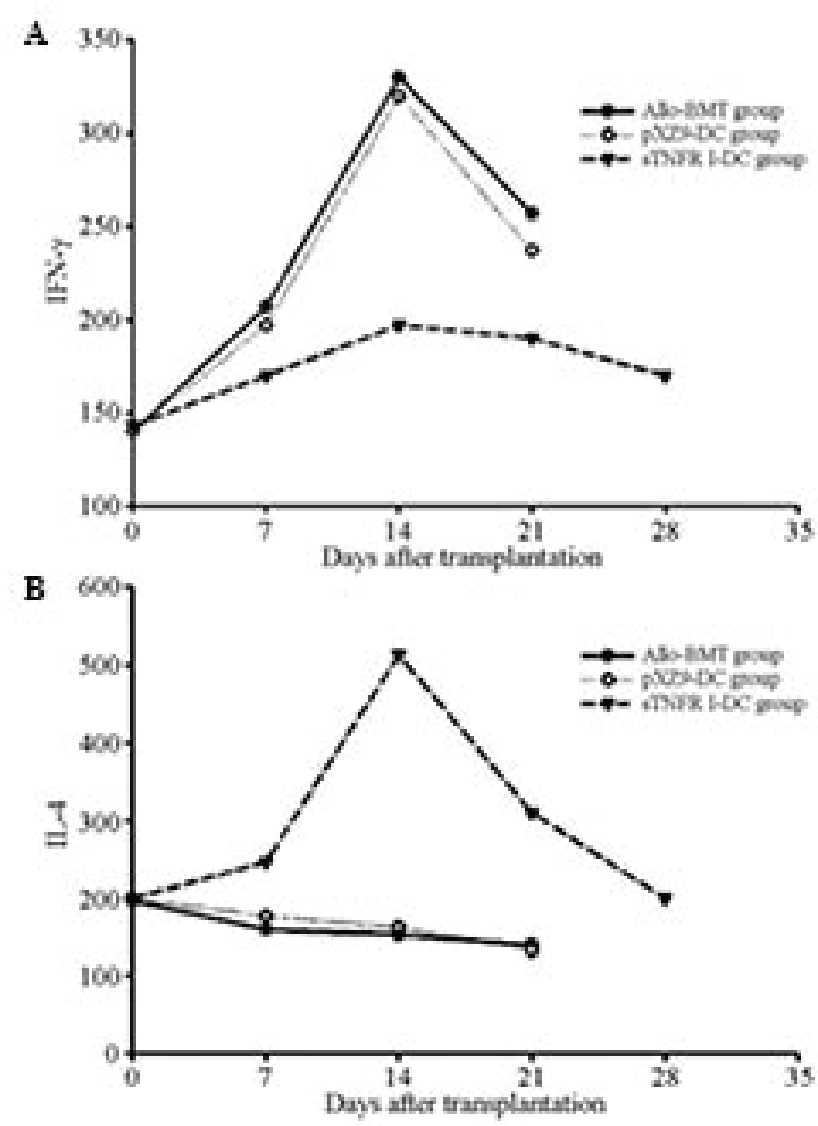

Figure 5. (A) Serum IFN- $\gamma$ and (B) IL-4 concentrations in mice at various time-points following transplantation. Allo-BMT, sTNFR1, soluble tumour necrosis factor receptor 1; allogeneic bone marrow transplantation; DC, dendritic cell; IFN, interferon; IL, interleukin.

pXZ9-imDC groups $(\mathrm{P}<0.05)$. The serum IFN- $\gamma$ concentration was also reduced in the $\mathrm{pXZ9-imDC}$ group compared with that of the allo-BMT group ( $\mathrm{P}<0.05)$ (Fig. 5A). The levels of IL-4 in the serum were reduced in the allo-BMT and pXZ9-imDC groups, but gradually increased in the sTNFR1-imDC group, peaking at $14 \mathrm{~d}$. There was significant difference in IL-4 levels between the sTNFR1-imDC and allo-BMT groups, and between the sTNFR1-imDC and pXZ9-imDC groups $(\mathrm{P}<0.05)$. No significant difference was detected between the allo-BMT and pXZ9-imDC group ( $\mathrm{P}>0.05$; Fig. 5B).

\section{Discussion}

At present, allo-BMT is regarded to be the most effective treatment for numerous malignant hematologic diseases, including leukemia, lymphoma and myelodysplastic syndrome $(24,25)$. The GVL effect exerted by donor T cells contributes to the efficacy of allo-BMT (26). However, GVHD is a life-threatening condition and over the past several decades, no existing method has been able to completely separate GVHD and GVL. Reducing the incidence and severity of GVHD whilst retaining the GVT effects is a crucial step in improving the overall effectiveness of allo-BMT against hematological malignancies (27-29). Several studies have indicated that the use of tolerogenic DCs or of freshly-isolated, regulatory T cells for the control of GVHD does not affect the activity of GVT against leukemic cells and lymphomas (30-32). Conventional therapies have previously targeted T cells; however, immunostimulatory DCs are critical to the pathogenesis of GVHD and also have tolerogenic properties (33). One therapeutic approach that has emerged is the use of imDCs for the induction of peripheral immune tolerance in allograft recipients. Administration of donor-provided imDCs or manipulation of imDCs using immunosuppressive cytokines, including IL-10 and TGF- $\beta$, significantly prolonged allograft survival in non-immunosuppressed transplant recipients $(7,34)$. Studies have indicated that intervention in DCs from recipients, donors or a third party may induce immunologic tolerance, which has an important function in the regulation of GVHD and GVL (35). The present study, in agreement with other studies, has demonstrated that unlike mature DCs which express high levels of MHC II and co-stimulatory molecules on their surface and induce immune responses, imDCs were deficient in co-stimulatory molecules, inhibited T-cell responses and induced tolerance. Moreover, further studies have suggested that imDCs expressing sTNFR1 were resistant to maturation induced by lipopolysaccharide stimulation and retained low levels of MHC II and co-stimulatory markers characteristic of imDCs. In the present study, donor imDCs were selectively manipulated by transfection with the $S T N F R 1$ gene to inhibit the maturation and activation of DCs. In leukemia mouse-models of allo-BMT, sTNFR1-modified imDCs induced long-lasting immune tolerance and ameliorated GVHD without affecting the GVL response.

The C57BL/6-derived T-cell leukemia/lymphoma cell line EL4 was used for leukemia modelling, as previously described (36), with minor modification. EL4 cells share histocompatibility antigens with normal C57BL/6 mice and the variation in the numbers of EL4 leukemia cells and normal target cells in the host, as well as tissue compensation, enable attenuation of GVHD and retention of GVL $(36,37)$. The GVL effect observed in this model depends on the alloreactivity of donor $\mathrm{T}$ cells which recognize various $\mathrm{MHC}$ molecules expressed on the surface of EL4 cells. In a previous study by our group, an EL4 H-2 ${ }^{\text {b }}$ leukemia model was constructed $(20,38)$ with a success rate of $100 \%$. The EL4 cells exhibited marked invasiveness and the leukemia model was rapid and stable with extensive infiltration into the liver and spleen. In addition to bone marrow transplantation, EL4 leukemia cells from C57BL/6 mice were infused, and mice in the leukemia group succumbed to leukemia within $18 \mathrm{~d}$ following transplantation. Anti-leukemia effects of allo-BMT were observed in the allo-BMT, pXZ9-imDC and sTNFR1-imDC groups. Few mice developed leukemia in these three groups and the survival rate and average survival duration were prolonged in the pXZ9-imDC and STNFR1-imDC groups compared with those of the allo-BMT group. Furthermore, the survival rate was prolonged in the sTNFR1-imDC group compared with that of the pXZ9-imDC group, suggesting that allo-BMT GVL effects were retained following pXZ9-imDC and sTNFR1-imDC infusions. Clinical scores were higher and GVHD was more severe in the allo-BMT group compared with the pXZ9-imDC and sTNFR1-imDC groups. The sTNFR1-imDC group produced stable chimeras and exhibited significantly fewer symptoms and a prolonged survival rate. The imDC infusion alone was able to attenuate GVHD. However, as the imDCs gradually matured due to antigen stimulation, 
the expression of co-stimulatory molecules increased, antigen presentation was enhanced and tolerance was reduced, which suppressed the ability of imDCs to inhibit GVHD. However, sTNFR1-imDCs attenuated GVHD and improved survival rates whilst maintaining imDC properties.

Developments in immunology and genetics have improved the understanding of the immunologic mechanisms of allo-hemopoietic stem cell transplantation. Recent evidence has indicated that GVHD is a process where T cells within donor transplants recognize 'foreign' recipient histocompatibility antigens, resulting in endogenous cytokine release and tissue over-reactivity. Consequently, $\mathrm{T}$ cells attack tissues expressing these antigens in a process known as cytokine storm $(39,40)$. $\mathrm{CD}^{+} \mathrm{T}$ cells have a critical function in the pathological process of initiating and amplifying GVHD (34). CD4 ${ }^{+} \mathrm{T}$ cells are categorized into Th1 and Th2 cells according to their active protein components. In murine models, donor $\mathrm{CD}^{+} \mathrm{Th} 1$ cells preferentially secrete type- 1 cytokines (IL- 2, IFN- $\gamma$ and TNF- $\alpha$ ) and induce significant GVHD, whereas donor Th2 cells which primarily secrete type-2 cytokines (IL-4, IL-5, IL-10 and IL-13), reduce GVHD and therefore downregulate the GVHD initiated by Th1/Tc1 cells $(3,39,41)$. In the present study, pXZ9-imDCs and sTNFR1-imDCs reduced the secretion of IFN- $\gamma$, a Th1 cytokine, and increased the production of IL-4, a Th2 cytokine, further indicating that pXZ9-imDCs and sTNFR1-imDCs stimulated immunologic tolerance of donor $\mathrm{CD}^{+} \mathrm{T}$ cells following activation of recipient antigens. Th1 cytokines function in immune activation, modulate cellular immune responses, enhance the activation and recruitment of macrophages, recruit B cells and promote cytotoxic T-cell proliferation to promote GVHD. By contrast, Th2 cytokines are associated with immunologic tolerance and reduce production of causative agents of GVHD (4,42).

pXZ9-imDCs and sTNFR1-imDCs induce immunologic tolerance by secreting cytokines that negatively regulate immunity, which is potentially a mechanism for immunologic tolerance induction (43). Variation between pXZ9-imDCs and sTNFR1-imDCs suggests that genetically modified DCs prevent induction of maturation by exogenous antigens and stabilize immunologic tolerance. However, half of the mice in the sTNFR1-imDC group succumbed to acute GVHD, revealing a marked limitation of sTNFR1-imDCs. This phenomenon may be due to a number of factors; for example, viral vectors are able to promote DC maturation, resulting in the gradual maturation of infused imDCs. In addition, recipient antigen-presenting cells participate in acute GVHD. In the present study, donor antigen-presenting cells were selectively manipulated; however, their activation was not completely inhibited. Therefore, multiple infusions for an extended duration or the concomitant use of immunosuppressants may improve therapeutic outcomes. Subsequent experiments are required to investigate the DC components in blood cells, distribution changes, homing and chemotaxis.

In conclusion, the EL4 leukemia/lymphoma model was successfully replicated in a mouse model. Infusion of sTNFR1-imDC prolonged survival, reduced expression levels of Th1 cytokines, increased expression levels of Th2 cytokines, significantly attenuated GVHD and maintained GVL effects in mice. The results of the present study therefore provided a novel insight into the clinical discrimination of GVL and GVHD and may therefore aid in the reduction of mortality following hematopoietic stem cell transplantation and leukemia relapse.

\section{Acknowledgements}

The present study was supported by the Medical Scientific Research Foundation of Jiangsu Province (grant no. 2014027), the Xuzhou Science and Technology Plan Program (grant no. XM13B040) and The Ordinary College Postgraduates Practice and Innovation Projects of Jiangsu Province in 2013-2014 (grant nos. CXLX13_99 and SJZZ_0200).

\section{References}

1. Mielcarek M, Storer B, Martin PJ, Forman SJ, Negrin RS, Flowers ME, Inamoto Y, Chauncey TR, Storb R, Appelbaum FR and Bensinger WI: Long-term outcomes after transplantation of HLA-identical related G-CSF-mobilized peripheral blood mononuclear cells versus bone marrow. Blood 119: 2675-2678, 2012.

2. Morecki S, YacovlevE, Gelfand Y, Shabat Y and Slavin S: Induction of graft-versus-leukemia (GVL) effect without graft-versus-host disease (GVHD) by pretransplant donor treatment with immunomodulators. Biol Blood Marrow Transplant 15: 406-415, 2009.

3. Edinger M, Powrie F and Chakraverty R: Regulatory mechanisms in graft-versus- host response. Biol Blood Marrow Transplant 15 (1 Suppl): 2-6, 2009

4. Huang Y, Feng S, Tang R, Du B, Xu K and Pan X: Efficacy of pretreatment of allografts with methoxypolyethylene glycol-succinimidyl-propionic acid ester in combination with an anti-OX40L monoclonal antibody in relieving graft-versus-host disease in mice. Int J Hematol 92: 609-616, 2010.

5. Sordi V and Piemonti L: Therapeutic plasticity of stem cells and allograft tolerance. Cytotherapy 13: 647-660, 2011.

6. Zhou F, Ciric B, Zhang GX and Rostami A: Immune tolerance induced by intravenous transfer of immature dendritic cells via up-regulating numbers of suppressive IL-10(+) IFN- $\gamma(+)$-producing CD4(+) T cells. Immunol Res 56: 1-8, 2013.

7. Cools N, Van Tendeloo VF, Smits EL, Lenjou M, Nijs G, Van Bockstaele DR, Berneman ZN and Ponsaerts P: Immunosuppression induced by immature dendritic cells is mediated by TGF-beta/IL-10 double-positive CD4+ regulatory T cells. J Cell Mol Med 12: 690-700, 2008.

8. Sun X, Jones HP, Dobbs N, Bodhankar S and Simecka JW: Dendritic cells are the major antigen presenting cells in inflammatory lesions of murine Mycoplasma respiratory disease. PLoS One 8: e55984, 2013.

9. Maldonado RA and von Andrian UH: How tolerogenic dendritic cells induce regulatory T cells. Adv Immunol 108: 111-165, 2010.

10. Fukaya T, Takagi H, Taya $\mathrm{H}$ and Sato K: DCs in immune tolerance in steady-state conditions. Methods Mol Biol 677: 113-126, 2011.

11. Trevejo JM, Marino MW, Philpott N, Josien R, Richards EC, Elkon KB and Falck-Pedersen E: TNF- $\alpha$-dependent maturation of local dendritic cells is critical for activating the adaptive immune response to virus infection. Proc Natl Acad Sci USA 98: 12162-12167, 2001.

12. Kleijwegt FS, Laban S, Duinkerken G, Joosten AM, Zaldumbide A, Nikolic T and Roep BO: Critical role for TNF in the induction of human antigen-specific regulatory $\mathrm{T}$ cells by tolerogenic dendritic cells. J Immunol 185: 1412-1418, 2010.

13. Jin JO, Park HY, Xu Q, Park JI, Zvyagintseva T, Stonik VA and Kwak JY: Ligand of scavenger receptor class A indirectly induces maturation of human blood dendritic cells via production of tumor necrosis factor-alpha. Blood 113: 5839-5847, 2009.

14. Bosè F, Raeli L, Garutti C, et al: Dual role of anti-TNF therapy: enhancement of TCR-mediated T cell activation in peripheral blood and inhibition of inflammation in target tissues. Clin Immunol 139: 164-176, 2011.

15. Baldwin HM, Ito-Ihara T, Isaacs JD and Hilkens CM: Tumour necrosis factor alpha blockade impairs dendritic cell survival and function in rheumatoid arthritis. Ann Rheum Dis 69: 1200-1207, 2010.

16. Rossol M, Meusch U, Pierer M, Kaltenhäuser S, Häntzschel H, Hauschildt $\mathrm{S}$ and Wagner U: Interaction between transmembrane TNF and TNFR $1 / 2$ mediates the activation of monocytes by contact with T cells. J Immunol 179: 4239-4248, 2007. 
17. Yi HJ and Lu GX: Adherent and non-adherent dendritic cells are equivalently qualified in GM-CSF, IL- 4 and TNF- $\alpha$ culture system. Cell Immunol 277: 44-48, 2012.

18. Xia Y, Dai J, Lu P, Huang Y, Zhu Y and Zhang X: Distinct effect of CD40 and TNF-signaling on the chemokine/chemokine receptor expression and function of the human monocyte-derived dendritic cells. Cell Mol Immunol 5: 121-131, 2008.

19. Huang YH, Chao YL, Tang RX, Wang SH, Zeng LY, Chen C, $\mathrm{Pan} X \mathrm{X}$ and $\mathrm{Xu} \mathrm{KL}$ : Lentivirus-mediated soluble tumor necrosis factor receptor 1 expression in mouse bone marrow-derived immature dendritic cells. J Clin Rehabil Tissue Eng Res 14 941-946, 2010

20. Cao J, Chen C, Zeng L, Li L, Li Z and Xu K: Engineered regulatory $\mathrm{T}$ cells prevent graft-versus-host disease while sparing the graft-versus-leukemia effect after bone marrow transplantation. Leuk Res 34: 1374-1382, 2010.

21. Cooke KR, Kobzik L, Martin TR, Brewer J, Delmonte J Jr, Crawford JM and Ferrara JL: An experimental model of idiopathic pneumonia syndrome after bone marrow transplantation: I. The roles of minor $\mathrm{H}$ antigens and endotoxin. Blood 88 : 3230-3239, 1996.

22. Asai O, Longo DL, Tian ZG, Hornung RL, Taub DD, Ruscetti FW and Murphy WJ: Suppression of graft-versus-host disease and amplification of graft-versus-tumor effects by activated natural killer cells after allogeneic bone marrow transplantation. J Clin Invest 101: 1835-1842, 1998.

23. SunK,WilkinsDE,AnverMR,Sayers TJ,Panoskaltsis-Mortari A, Blazar BR, Welniak LA and Murphy WJ: Differential effects of proteasome inhibition by bortezomib on murine acute graft-versus-host disease (GVHD): delayed administration of bortezomib results in increased GVHD-dependent gastrointestinal toxicity. Blood 106: 3293-3299, 2005.

24. Appelbaum FR: Haematopoietic cell transplantation as immunotherapy. Nature 411: 385-389, 2001.

25. Konuma T, Kato S, Ooi J, Oiwa-Monna M, Kawamata T, Tojo A and Takahashi S: Comparable long-term outcome of unrelated cord blood transplantation with related bone marrow or peripheral blood stem cell transplantation in patients aged 45 years or older with hematologic malignancies after myeloablative conditioning. Biol Blood Marrow Transplant 20 1150-1155, 2014

26. van Besien K: Allogeneic transplantation for AML and MDS GVL versus GVHD and disease recurrence. Hematology Am Soc Hematol Educ Program 2013: 56-62, 2013.

27. Sinkovics JG: Antileukemia and antitumor effects of the graft-versus-host disease: a new immunovirological approach. Acta Microbiol Immunol Hung 57: 253-347, 2010.

28. Kim HJ, Min WS, Cho BS, Eom KS, Kim YJ, Min CK, Lee S, Cho SG, Jin JY, Lee JW and Kim CC: Successful prevention of acute graft-versus-host disease using low-dose antithymocyte globulin after mismatched, unrelated, hematopoietic stem cell transplantation for acute myelogenous leukemia. Biol Blood Marrow Transplant 15: 704-717, 2009.
29. Capitini CM, Herby S, Milliron M, Anver MR, Mackall CL and Fry TJ: Bone marrow deficient in IFN-\{gamma\} signaling selectively reverses GVHD-associated immunosuppression and enhances a tumor-specific GVT effect. Blood 113: 5002-5009, 2009.

30. Chorny A, Gonzalez-Rey E, Fernandez-Martin A, Ganea D and Delgado M: Vasoactive intestinal peptide induces regulatory dendritic cells that prevent acute graft-versus-host disease while maintaining the graft-versus-tumor response. Blood 107: 3787-3794, 2006

31. Stenger EO, Turnquist HR, Mapara MY and Thomson AW: Dendritic cells and regulation of graft-versus-host disease and graft-versus-leukemia activity. Blood 119: 5088-5103, 2012.

32. Semple K, Yu Y, Wang D, Anasetti C and Yu XZ: Efficient and selective prevention of GVHD by antigen-specific induced Tregs via linked-suppression in mice. Biol Blood Marrow Transplant 17: 309-318, 2011.

33. Toubai T, Mathewson N and Reddy P: The role of dendritic cells in graft-versus-tumor effect. Front Immunol 5: 66, 2014.

34. Sun W, Wang Q, Zhang L, Pan J, Zhang M, Lu G, Yao H, Wang J, Cao X: TGF-beta(1) gene modified immature dendritic cells exhibit enhanced tolerogenicity but induce allograft fibrosis in vivo. J Mol Med (Berl) 80: 514-523, 2002.

35. Sela U, Olds P, Park A, Schlesinger SJ and Steinman RM: Dendritic cells induce antigen specific regulatory $\mathrm{T}$ cells that prevent graft versus host disease and persist in mice. J Exp Med 208: 2489-2496, 2011.

36. Yang YG, Sergio JJ, Pearson DA, Szot GL, Shimizu A and Sykes M: Interleukin-12 preserves the graft-versus-leukemia effect of allogeneic CD8 T cells while inhibiting CD4-dependent graft-versus-host disease in mice. Blood 90: 4651-4660, 1997

37. Mapara MY and Sykes M: Induction of mixed vs full chimerism to potentiate GVL effects after bone-marrow transplantation. Methods Mol Med 109: 469-474, 2005.

38. Li YJ, Cao J, Chen C, et al: Establishment and identification of mouse lymphoma cell line EL4 expressing red fluorescent protein. J Exp Hematol 18: 107-110, 2010 (In Chinese).

39. Ball LM and Egeler RM; EBMT Paediatric Working Party: Acute GvHD: pathogenesis and classification. Bone Marrow Transplant 41 (Suppl 2): S58-S64, 2008.

40. Sun Y, Tawara I, Toubai T and Reddy P: Pathophysiology of acute graft-versus-host disease: recent advances. Transl Res 150: 197-214, 2007.

41. Coghill JM, Sarantopoulos S, Moran TP, Murphy WJ, Blazar BR and Serody JS: Effector CD4+ T cells, the cytokines they generate, and GVHD: something old and something new. Blood 117: 3268-3276, 2011.

42. Li JM, Giver CR, Lu Y, Hossain MS, Akhtari M and Waller EK: Separating graft-versus-leukemia from graft-versus-host disease in allogeneic hematopoietic stem cell transplantation. Immunotherapy 1: 599-621, 2009.

43. Li X, Yang A, Huang H, et al: Induction of type $2 \mathrm{~T}$ helper cell allergen tolerance by IL-10-differentiated regulatory dendritic cells. Am J Respir Cell Mol Biol 42: 190-199, 2010. 